\title{
Resonance search for new physics in the photon+jet final state at $13 \mathrm{TeV}$
}

\author{
Varun Sharma* \\ University of Delhi, India \\ on behalf of the CMS Collaboration \\ E-mail: varun.sharma@cern.ch
}

\begin{abstract}
A search is presented for excited quarks $\left(q^{*}\right)$ decaying into $\gamma+$ jet final state in $p p$ collisions at $\sqrt{s}=13 \mathrm{TeV}$. The search is done with the data taken by the CMS experiment at the CERN LHC in 2015 corresponding to an integrated luminosity of $2.7 \mathrm{fb}^{-1}$. Results are presented in terms of 95\% CL upper limits on cross section times branching fraction as a function of excited quark mass $\left(M_{q^{*}}\right)$. Limits on excited quarks are presented as a function of their mass and coupling strength; masses below $4.37 \mathrm{TeV}$ are excluded at $95 \% \mathrm{CL}$ for coupling multipliers $f=1.0$.
\end{abstract}

38th International Conference on High Energy Physics 3-10 August 2016

Chicago, USA

${ }^{*}$ Speaker. 


\section{Introduction}

This document reports a search for excited quarks in events containing a photon and a jet in the final state. The search is motivated to answer some of the open questions within the standard model of particle physics. Standard model, despite its success, is conceived as an effective Lagrangian to a more fundamental theory. Several extensions of the standard model predict quark substructure or composite quarks [1].

A compelling signature for substructure of quarks would be the discovery of an excited state of a quark $\left(q^{*}\right)$. This analysis searches for the single process $q g \rightarrow q^{*} \rightarrow q g$. The signal model considered includes the dominant production modes of excited quarks $\left(u^{*}\right.$ and $\left.d^{*}\right)$ with spin $-\frac{1}{2}$. The compositeness scale and mass of the excited quarks are set to be equal in the signal model, i.e., $\Lambda=M_{q^{*}}$. The search is based on proton-proton collision data collected by the CMS experiment [2] at $\sqrt{s}=13 \mathrm{TeV}$ in 2015, corresponding to an integrated luminosity of $2.7 \mathrm{fb}^{-1}$.

\section{Event Selection}

The data sample used in the analysis consists of events that pass a trigger with a threshold of $165 \mathrm{GeV}$ on the transverse momentum of the photon. In each event, photons with $p_{\mathrm{T}}^{\gamma}>190 \mathrm{GeV}$ and $\left|\eta^{\gamma}\right|<1.4442$, are selected in the list of the possible photon candidates. The photon with highest transverse momentum (leading) amongst the ones which satisfy the photon identification and isolation requirements [3], is used as the final photon candidate in the event.

The jets separated from the selected photon candidate by $\Delta R>0.5$ and satisfying a stringent jet identification criteria [3] are selected as the possible jet candidates. The jet is required to be within the pseudorapidity region $\left|\eta^{\text {jet }}\right|<2.4$ and must have a transverse momentum $p_{\mathrm{T}}^{\text {jet }}>190 \mathrm{GeV}$. If more than one photon or jet candidates exist in the event, the $\gamma+$ jet invariant mass is calculated using the leading photon candidate and leading jet candidate.

To reduce the backgrounds from t-channel processes, $\mid \Delta \eta(\gamma$, jet $) \mid<1.8$ selection is imposed between the selected photon and the jet candidate while keeping large acceptance for the signal. Events with the photon and jet back-to-back in $\phi, \mid \Delta \phi(\gamma$,jet $) \mid>1.5$, are kept. The invariant mass of the $\gamma+$ jet system is required to be $M_{\gamma, \text { jet }}>695 \mathrm{GeV}$ to have full kinematic acceptance for events with the photon and jet $p_{\mathrm{T}}, \eta$ and $|\Delta \eta|$ requirements. The signal efficiency goes from about $49 \%$ at $M_{q^{*}}=1 \mathrm{TeV}$ to $58 \%$ at $M_{q^{*}}=2 \mathrm{TeV}$ and $60 \%$ at $M_{q^{*}}=5 \mathrm{TeV}$.

\section{Background Fit and Signal Modeling}

To search for $\gamma+$ jet resonances, the non-resonant standard model $\gamma+$ jet spectrum is modelled using a smooth parameterization, $\frac{d \sigma}{d m}=\frac{p_{0}(1-m / \sqrt{s})^{p_{1}}}{(m / \sqrt{s})^{p_{2}+p_{3} \ln (m / \sqrt{s})}}$, where $p_{0}, p_{1}, p_{2}$, and $p_{3}$ are the four parameters used to describe the background. The parametric coefficients are obtained from a fit to the data events and considered as nuisance parameters in the hypothesis testing, providing a data-driven estimation for the shape of the background spectrum. The bias in the fit function is studied using MC simulations. The mass distribution shown in Fig 1 is binned with bin-width approximately equal to the expected $\gamma+$ jet mass resolution, which varies from about $4.5 \%$ at a mass of $1 \mathrm{TeV}$ to $3.6 \%$ at $4 \mathrm{TeV}$. The highest mass event observed in data is at $3.29 \mathrm{TeV}$. 
The signal distribution for excited quarks is simulated with PYTHIA8 event generator. The natural width of the resonance peak, at parton level before experimental reconstruction, can be approximated as $\Gamma \sim 0.03 f^{2} M_{q^{*}}$. The production cross section is also proportional to $f^{2}$.

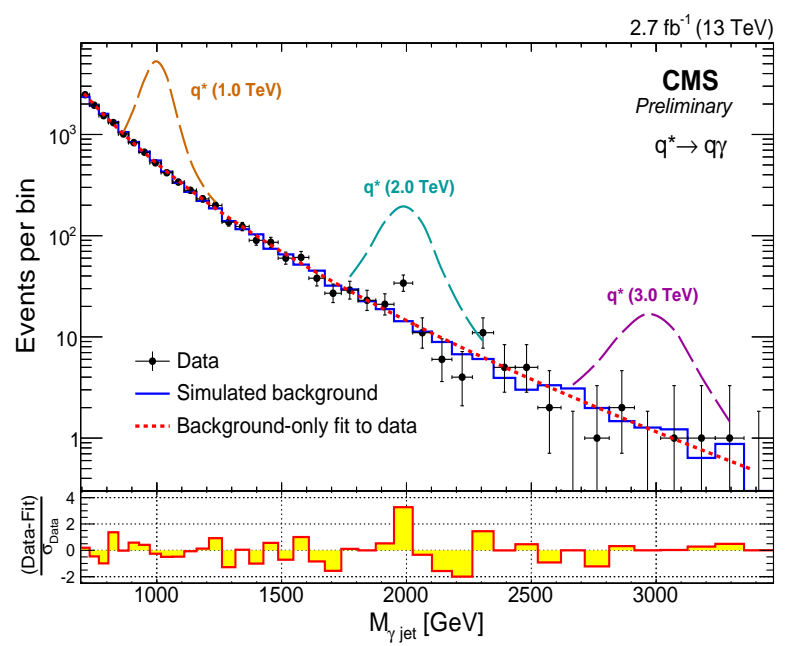

Figure 1: The $\gamma+$ jet invariant mass distribution in data (points) and MC (histogram) prediction after full selection. The result of the fit to the data is shown with the red dotted curve. Simulations of $q^{*}$ signals for coupling multiplier $f=1.0$ are shown at masses of 1,2 and $3 \mathrm{TeV}$ (dashed curves).

\section{Results}

The sources of systematic uncertainties affecting only signal are listed in Table 1 and for the background shape uncertainty, the background parameters are marginalized with a flat prior. A

\begin{tabular}{lc}
\hline Major sources & Uncertainty (\%) \\
\hline Jet energy resolution (JER) & 10 \\
Photon energy resolution (PER) & 0.5 \\
Jet energy scale (JES) & $0.5-0.8$ \\
Photon energy scale (PES) & 1.0 \\
Photon identification \& isolation & $2.0-4.0$ \\
Luminosity & 2.7 \\
\hline
\end{tabular}

Table 1: Major sources of systematic uncertainty affecting signal.

Bayesian formalism using a binned likelihood with a uniform prior for the signal cross section is used for estimating the 95\% confidence level (CL) upper limit on the cross section. Log-normal prior distribution functions are used to model the systematic uncertainties which are treated as nuisance parameters to evaluate the limits.

The $95 \% \mathrm{CL}$ upper limits on $\sigma \times \mathscr{B}$ as a function of $M_{q^{*}}$ is shown in Fig 2a. A lower bound of $3.5(2.9) \mathrm{TeV}$ on the mass of excited quarks is obtained for $f=1.0(0.5)$ by comparing the observed limits with the leading order theoretical predictions. The results shown in Fig $2 \mathrm{~b}$ presents limits on the excited quark mass as a function of compositeness scale. 


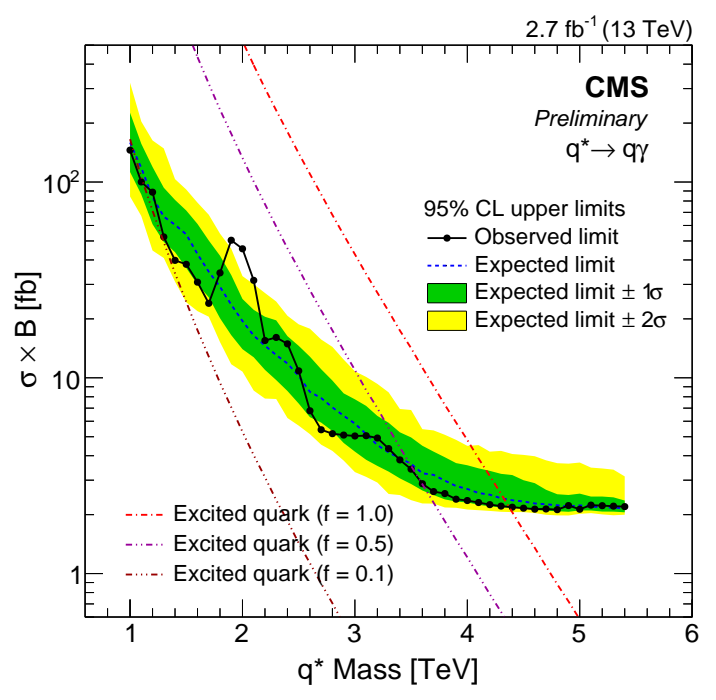

(a) The expected and observed 95\% CL upper limits on $\sigma \times \mathscr{B}$ for excited quark search in $\gamma+$ jet final state corresponding to coupling $f=1.0$. The limits are also compared with the theoretical predictions for excited quark production, shown for coupling values $f=1.0,0.5$, and 0.1 . The uncertainty at $1 \sigma$ and $2 \sigma$ levels are shown as green and yellow shaded bands around expected limit.

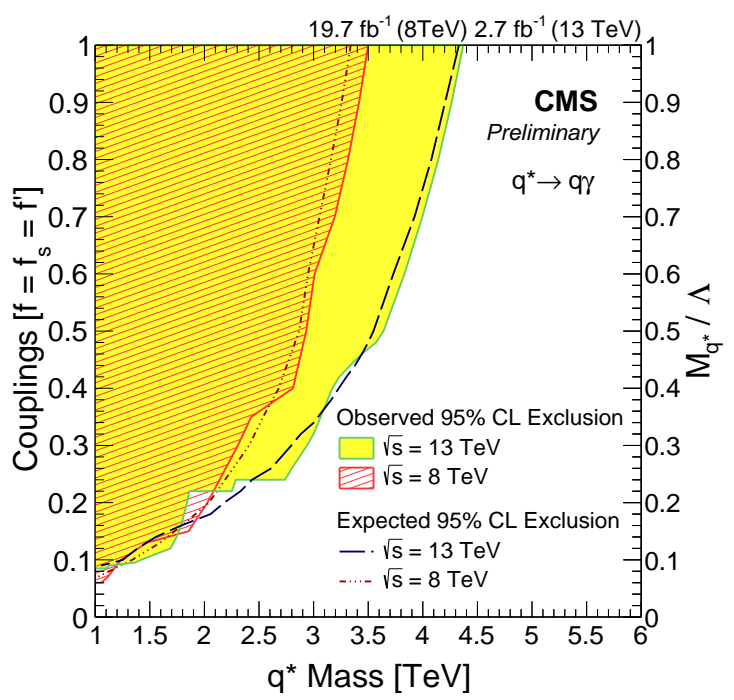

(b) The observed (yellow filled) and expected (blue dashed line) excluded regions at $95 \% \mathrm{CL}$ as a function of excited quark mass and the coupling strength for $\Lambda=M_{q^{*}}$ (left axis) or the mass divided by compositeness scale for coupling strength of $f=1.0$ (right axis). The excluded region from Run-I is also shown (red shaded).

Figure 2

\section{Summary}

A search for excited quarks in the $\gamma+$ jet final state has been performed, using $2.7 \mathrm{fb}^{-1}$ of proton-proton collision data collected with the CMS experiment at $\sqrt{s}=13 \mathrm{TeV}$. The data are found to be consistent with the predictions of the standard model and no evidence is found for an excited quark resonance. A $95 \%$ CL upper limit is placed on $\sigma \times \mathscr{B}$ for $q^{*}$ production in the $\gamma+$ jet final state.

Comparing the upper limits on $\sigma \times \mathscr{B}$ with the theoretical predictions for $q^{*}$, an excited quark state with masses in range $1.0<M_{q^{*}}<4.37 \mathrm{TeV}$ are excluded at $95 \% \mathrm{CL}$ for $f=1.0$. Also, excited quarks with masses $1.0<M_{q^{*}}<3.64(1.36) \mathrm{TeV}$ are excluded for $f=0.5(0.1)$. We also present the excluded mass as a function of coupling strength in Fig $2 b$.

\section{References}

[1] U. Baur, I. Hinchliffe, D. Zeppenfeld, Excited quark production at hadron colliders, Int. J. Mod. Phys. A 2 (1987) 1285.

[2] CMS Collaboration, The CMS experiment at the CERN LHC, J. Instrum. 3 (2008) S08004.

[3] CMS Collaboration, Search for excited quarks in the $\gamma+$ jet final state in proton-proton collisions at $\sqrt{s}=13 \mathrm{TeV}$, CMS PAS EXO-16-015. 